\title{
Normalizing the Neural Correlates of Binge-Eating Disorder with Lisdexamfetamine: A Case Report and Literature Review
}

\author{
Leyla R. Brucar ${ }^{1 *}$, Amna Hyder ${ }^{2}$, Carl Laird Birmingham ${ }^{3 *}$ \\ ${ }^{1}$ University of British Columbia, Vancouver, BC, Canada \\ ${ }^{2}$ Masters Program in Neuroscience, University of British Columbia, Vancouver, BC, Canada \\ ${ }^{3}$ Department of Psychiatry, University of British Columbia, Vancouver, BC, Canada \\ Email: *leyla.brucar@alumni.ubc.ca, ${ }^{*}$ carl.birmingham@ubc.ca
}

How to cite this paper: Brucar, L.R., Hyder, A. and Birmingham, C.L. (2018) Normalizing the Neural Correlates of Binge-Eating Disorder with Lisdexamfetamine: A Case Report and Literature Review. Open Journal of Psychiatry, 8, 142-151.

https://doi.org/10.4236/ojpsych.2018.82014

Received: March 1, 2018

Accepted: April 21, 2018

Published: April 24, 2018

Copyright (C) 2018 by authors and Scientific Research Publishing Inc. This work is licensed under the Creative Commons Attribution International License (CC BY 4.0).

http://creativecommons.org/licenses/by/4.0/

\begin{abstract}
The brain regions associated with binge-eating disorder (BED) and those targeted by Lisdexamfetamine (LDX) overlap. The present paper reviews the psychobehavioural and neuropharmacological mechanisms at play in BED and compulsive eating behaviours, along with the mechanisms of action LDX is thought to play. We present one case of chronic BED and highlight the EEG scans pre- and post-LDX treatment. The normalization of the patient's electroencephalogy (EEG) activity associated with BED after LDX supports theorized mechanisms of LDX action, further explaining its efficacy in treatment. A significant decrease in theta band power was observed in the patient after treatment with LDX. Specifically, this decrease was modulated by changes in the F8 electrode corresponding to the orbitofrontal cortex (OFC) - An area associated with deficits in inhibitory function, seen in addictions and BED. A better understanding of the psychobehavioural and neuropharmacological mechanisms involved in BED and LDX treatment has the potential to significantly enhance our knowledge of the underlying pathogenesis, potential prevention, and optimal treatment options for patients.
\end{abstract}

\section{Keywords}

Binge-Eating Disorder, Lisdexamfetamine, Brain Mechanisms, Compulsive Eating, EEG, LORETA, Power Spectral Density, Theta Band

\section{Introduction}

Binge-eating disorder (BED), a serious public health issue, has been gaining recognition as the most widespread of the eating disorder types [1] [2]. BED is 
described as recurrent episodes of particularly large amounts of food intake within a discrete period of time, lack of control over consumption, marked psychological distress and physical discomfort, along with an absence of compensatory behaviours after the occurrence of the binge episodes [3]. As of 2013, BED has been included as an official diagnosis in the main section of the Diagnostic and Statistical Manual of Mental Disorders, Fifth Edition (DSM-V).

In 2015, the use of the d-amphetamine prodrug Lisdexamfetamine dimesylate (LDX), more commonly known as Vyvanse, received approval from the US Food and Drug Administration (FDA) for the treatment of BED. It was initially specified for the treatment of Attention Deficit Hyperactivity Disorder (ADHD), and later was proven to also have efficacy in limiting the occurrence of binge-eating behaviours [1] [4] [5] [6] [7].

The following is a case report that looks at the brain data of a patient with BED, both before and after starting her treatment with LDX. Data is in line with previous studies on the pathways involved in BED, and the efficacy of using LDX to treat BED and compulsive eating behaviours. This case report highlights brain mechanisms and regions implicated in BED, and possible mechanisms of LDX action.

\section{Mechanisms of Binge-Eating Disorder and Mechanisms of Action in Lisdexamfetamine}

Data suggests that many psychobehavioural and neuropharmacological mechanisms are at play in BED and associated compulsive behaviour. Moore et al. [8] [9] explained that the transdiagnostic construct of compulsive eating seen in BED and other conditions, can be broken down into three behavioural elements: habitual overeating, overeating to relieve a negative emotional state, and overeating despite adverse consequences. Each element's dysfunction can be mapped onto the brain to find relevant regions that are at play. Habitual overeating involves dysfunction in brain areas implicated in associative learning, reinforcement, and habit forming: the basal ganglia, ventral striatum, and dorsolateral striatum (DLS), respectively [8] [9]. Changes in BED habit forming are hypothesized to be due to a shift in dopaminergic signaling from the repeated activation in the ventral striatum following presentation of palatable food stimulus, to the more goal directed, habit forming region of the DLS [2] [8].

Similar to symptoms of Obsessive Compulsive Disorder (OCD), another element involves engaging in behaviours that promote negative reinforcement to relieve emotional states [8] [9] [10]. Engagement in compulsive eating behaviours promotes relief from anxiety, stress, and distress [8] [9]. The neurobiological processes involved in the second element include the desensitization of the mesocorticolimbic dopaminergic system, and neuroadaptations in the brains stress system in the extended amygdale, similar to that of someone withdrawing from drugs and alcohol [8] [9] [11]. For compulsive eating behaviours in BED, continued intake of palatable food becomes necessary to prevent experiencing negative emotional states [11]. 
Overeating despite adverse consequences, the last element of compulsive eating behaviour described by Moore et al., concerns the loss of control over food intake [8] [9]. This loss of control is exemplified by the inability to cease food consumption once started, despite feeling negative physical and psychological results [3] [8] [9]. Similar to the circuits implicated in addiction, these deficits of inhibitory control are found in the prefrontal cortex (PFC) and are meant to suppress inappropriate and dangerous actions [12] [13]. Hyperactivity of the dorsolateral PFC (dlPFC), anterior cingulate (ACC), and orbitofrontal (OFC) cortices, and hypoactivity of the ventromedial PFC (vmPFC) are thought to be at the route of compulsivity in binge-eating behaviours [8] [9].

In terms of neuropharmacology, three systems will be highlighted, the dopamine (DA), trace amine-associated receptor-1, and the serotonin (5-HT) systems. The dopaminergic pathways are implicated in the motivational aspects of compulsive eating and BED. The signal dominance of the Dopamine Type- 1 Receptor neurons (D1R) found in the DLS of those with binge-eating behaviour showed increased habit-forming functioning when triggered by palatable foods [14]. Overstimulation of these dopaminergic pathways were found to bring about insensitivity to the outcomes of these habitual actions surrounding food intake, and thus contributed to the loss of pleasure, and deficits in the motivation that cause action [14]. As a result, compulsive eating behaviours develop as a form of symptom relief in order to combat the feelings of anhedonia and motivational deficits [8] [9] [14]. Furlong et al., found that when a D1R agonist was injected into the dorsolateral striatum, the habit-forming performance initially seen was inhibited, and normal goal directed functioning and sensitivity to palatable foods was restored [14]. Backed by several studies that show decreased DA activity in addictive behaviour, the loss of inhibitory control exhibited by those with BED and compulsive eating behaviours was found to be attributed to vulnerabilities of DA signaling in the PFC [8] [9].

Evidence suggests that the trace amine-associated receptor-1 (TAAR1) plays a vital role in the regulation of behaviours associated with drugs of abuse, and the compulsive eating behaviours found in BED. TAAR1 is a G-protein coupled receptor activated by trace amines and neurotransmitters like DA and 5-HT, and has been found to significantly influence DA signaling in the mesocorticolimbic system: the reward pathways [15]. A lack of TAAR1 within the reward pathways results in heightened DA firing and thus, increases reinforcement and the reward effects of drugs of abuse and addictive behaviours [15]. Contrarily, TAAR1 activation reduces DA firing, resulting in the suppression of addictive behaviours [15]. It was found in a study conducted on binge-eating in rats, that binge-eating behaviours activated by palatable foods decreased TAAR1 protein levels in PFC pathways, and the TAAR1 agonist RO5256390, decreased compulsive eating behaviours [15]. As palatable food intake has been found to activate the reward circuit and release DA in PFC pathways, the modulation of TAAR1 in these areas have been proposed as a remedial option for addictive disorders including BED [8] [15]. 
Research has suggested links between dysfunction of 5-HT neurotransmitters, mood regulation and binge-eating behaviours [8] [16] [17]. Impaired 5-HT synaptic transmission indicated by reduced 5-HT content found in the brain following 5-HT uptake, was linked to the development of binge-eating in humans [16]. As a result, 5-HT inhibitors have been proposed as potential therapeutics, which will help in reducing the frequency of binge-eating behaviours and symptoms [4] [8] [18].

Evidence has shown LDX to specifically target psychobehavioural and neuropharmacological mechanisms at play in BED [8]. This prodrug works through the modulation of monoamine transmission, including DA and 5-HT [7] [8] [19]. After administration in rats, LDX was found to increase sustained DA in the striatum [8]. This increase in striatal DA is thought to recover the low DA states characteristic of those with compulsive eating behaviours, and in turn, provide relief from the negative emotional states experienced by those with BED [8] [20]. Furthermore, LDX improved inhibitory control deficits through increasing the extracellular concentration of DA in the PFC [8] [20]. The active metabolite in LDX, d-amphetamine, also activates TAAR1 [8]. This activation is another potential mechanism for why LDX is able to restore impaired prefrontal functioning in $\mathrm{BED}$ [8] [16]. In addition, this d-amphetamine inhibits monoamine reuptake increasing 5-HT release and concentration in the striatum [6] [8] [19]. Via LDX and its resulting restoration of 5-HT activity, a reduction of compulsive eating behaviours in those with BED is seen [6] [8] [19].

\section{Case Description}

A 56-year old female patient began seeking treatment from the attending physician after a 45-year history with BED. Her first episode of binge-eating was reported at the age of 11 and had continued without remission ever since. Her medical history includes a cholecystectomy at the age of 17, a roux-en-y gastric bypass surgery at 41 years of age, and gastric band surgery a few years following. She also underwent a partial hysterectomy in the past and has Factor V Leiden deficiency. Family history: no noted disorders; patient habits: nonsmoker, no alcohol consumption.

Before binge episodes-which occurred up to three times a day without compensatory purging behaviours, she reported having the feeling of being spaced out, and when the binge began, feeling out of control and eating about three times the volume of a normal meal. She reported that years of binge-eating resulted in low self-esteem and had greatly interfered with her life. She denied symptoms of depression, anxiety, OCD, hallucinations, delusions, and other comorbidities, and was previously tried on Zoloft and Citalopram but reported no benefit or cessation of binge-eating symptoms. Further, she tried psychotherapy on a number of occasions without any benefit.

On examination, her ears were clear, optic fundi were normal, nose was clear, mouth was clear, and thyroid was within normal limits. Her chest was clear, and 
heart sounds were normal with no extra sounds or murmurs. There were no masses, bruits, tenderness or organomegaly of the abdomen, and extremities were normal. She did not have a Trousseau or Chvostek's sign.

After examination, the attending physician recommended Low Resolution Electromagnetic Tomography Analysis (LORETA) neurofeedback and LDX. After five sessions with neurofeedback, the patient agreed to try LDX at an initial $30 \mathrm{mg}$ a day for 6 months, with the potential to increase the dosage to $50 \mathrm{mg}$ and $70 \mathrm{mg}$ if necessary. Along with LDX, a $25 \mathrm{mg}$ a day dose of Zoloft was prescribed. Other medications she was taking were $88 \mathrm{mg}$ a day of Aspirin for Factor V Leiden deficiency, and iron in the form of Palafer and a multivitamin.

\section{Analysis}

Electroencephalogy (EEG) LORETA scans were recorded using a 21 channel NeXus cap at resting state both before and 3 months after treatment with LDX. LORETA Progress Report was used to generate Z-scores by comparing activity in each region and frequency band to a database based on the age and gender of the participant. The most deviant Z-Scores for each Brodmann pair (>99\% variance) are highlighted in red in Figure 1.

At low and high frequencies, ocular and muscle artifacts are orders of magnitudes higher than neural signals. Although artifact reduction methods do exist, they are difficult to implement in smaller sample sizes. Thus, the data was filtered between $2-40 \mathrm{~Hz}$ and additional artifacts were removed by manual inspection and Independent Component Analysis (ICA). The cleaned data was fast

Initial:

\begin{tabular}{|l|r|r|r|r|r|r|r|r|r|r|}
\hline BA1 & Delta & Theta & Alpha & Alpha1 & Alpha2 & Beta & Beta1 & Beta2 & Beta3 & Hbeta \\
\hline 13 L & 5.72 & 6.1 & 3.93 & 3.54 & 4.12 & 2.59 & 4.21 & 3.19 & 1.63 & 0.99 \\
\hline 13 R & 5.69 & 6.46 & 4.63 & 4.04 & 5.05 & 2.78 & 4.59 & 3.1 & 1.79 & 1.11 \\
\hline 24 L & 3.66 & 4.82 & 3.21 & 2.83 & 3.59 & 1.66 & 3.06 & 1.84 & 0.92 & 0.57 \\
\hline 24 R & 3.66 & 4.82 & 3.22 & 2.83 & 3.6 & 1.67 & 3.08 & 1.85 & 0.93 & 0.57 \\
\hline 25 L & 3.79 & 5.11 & 3.43 & 3.15 & 3.76 & 1.64 & 2.99 & 1.8 & 0.94 & 0.6 \\
\hline 25 R & 3.92 & 5.36 & 3.71 & 3.36 & 4.1 & 1.75 & 3.25 & 1.91 & 0.97 & 0.61 \\
\hline 32 L & 4.26 & 5.24 & 3.84 & 3.59 & 4.24 & 1.78 & 3.12 & 1.96 & 1.07 & 0.71 \\
\hline 32 R & 4.29 & 5.31 & 4.06 & 3.76 & 4.52 & 1.85 & 3.3 & 2.03 & 1.08 & 0.73 \\
\hline 34 L & 4.85 & 5.79 & 3.93 & 3.39 & 4.18 & 2.36 & 4.04 & 2.72 & 1.44 & 0.93 \\
\hline 34 R & 5.35 & 6.51 & 4.45 & 3.79 & 4.87 & 2.71 & 4.6 & 3.02 & 1.67 & 1.09 \\
\hline 44 L & 5.19 & 7.29 & 5.82 & 5.61 & 5.84 & 2.56 & 4.52 & 2.83 & 1.53 & 0.97 \\
\hline 44 R & 5.15 & 7.46 & 6.33 & 6.2 & 6.56 & 2.76 & 4.82 & 3 & 1.66 & 1.1 \\
\hline 45 L & 5.16 & 7.24 & 5.83 & 5.62 & 5.86 & 2.55 & 4.51 & 2.81 & 1.53 & 0.98 \\
\hline 45 R & 5.16 & 7.46 & 6.36 & 6.21 & 6.6 & 2.81 & 4.85 & 3.04 & 1.7 & 1.15 \\
\hline 46 L & 4.3 & 6.04 & 5.19 & 5.08 & 5.16 & 2.46 & 4.34 & 2.78 & 1.45 & 1.02 \\
\hline 46 R & 4.79 & 6.98 & 5.66 & 5.7 & 5.6 & 2.45 & 4.37 & 2.7 & 1.43 & 0.91 \\
\hline 47 L & 5.07 & 6.69 & 5.23 & 4.91 & 5.36 & 2.47 & 4.3 & 2.73 & 1.51 & 0.97 \\
\hline 47 R & 5.26 & 7.41 & 6.22 & 5.88 & 6.55 & 2.91 & 5.02 & 3.14 & 1.78 & 1.2 \\
\hline la L & 5.04 & 5.72 & 3.69 & 3.17 & 3.91 & 2.43 & 4.07 & 2.86 & 1.52 & 0.96 \\
\hline la R & 5.34 & 6.17 & 3.93 & 3.3 & 4.33 & 2.69 & 4.46 & 3.03 & 1.7 & 1.1 \\
\hline us L & 5.03 & 5.41 & 3.23 & 2.74 & 3.43 & 2.38 & 3.89 & 2.87 & 1.51 & 0.95 \\
\hline us R & 5.21 & 5.67 & 3.36 & 2.8 & 3.71 & 2.56 & 4.13 & 2.97 & 1.65 & 1.05 \\
\hline
\end{tabular}

\section{Final:}

\begin{tabular}{|l|r|r|r|r|r|r|r|r|r|r|}
\hline BA1 & Delta & Theta & Alpha & Alpha1 & Alpha2 & Beta & Beta1 & Beta2 & Beta3 & Hbeta \\
\hline 13 L & 4.9 & 5.02 & 3.09 & 2.98 & 3.11 & 1.45 & 2.88 & 1.74 & 0.67 & 0.11 \\
\hline 13 R & 1.41 & 1.83 & 1.02 & 0.96 & 1.07 & 0.18 & 0.82 & 0.21 & -0.14 & -0.12 \\
\hline 24 L & 2.68 & 3.23 & 1.74 & 1.64 & 1.8 & 0.33 & 1.32 & 0.41 & -0.17 & -0.35 \\
\hline $\mathbf{2 4}$ R & 2.64 & 3.19 & 1.7 & 1.61 & 1.77 & 0.32 & 1.29 & 0.39 & -0.18 & -0.35 \\
\hline 25 L & 2.86 & 3.55 & 1.99 & 1.95 & 2.03 & 0.36 & 1.36 & 0.42 & -0.15 & -0.35 \\
\hline 25 R & 2.36 & 3.03 & 1.59 & 1.55 & 1.62 & 0.2 & 1.09 & 0.25 & -0.25 & -0.36 \\
\hline 32 L & 3.39 & 3.92 & 2.58 & 2.57 & 2.66 & 0.62 & 1.77 & 0.67 & 0.03 & -0.23 \\
\hline 32 R & 3.01 & 3.49 & 2.31 & 2.29 & 2.4 & 0.5 & 1.57 & 0.53 & -0.05 & -0.25 \\
\hline 34 L & 4.51 & 5.2 & 3.33 & 3.03 & 3.42 & 1.56 & 3.13 & 1.8 & 0.71 & 0.19 \\
\hline 34 R & 1.79 & 2.37 & 1.26 & 1.08 & 1.38 & 0.47 & 1.28 & 0.56 & 0.04 & 0.02 \\
\hline $\mathbf{4 4 ~ L}$ & 4.6 & 6.27 & 4.79 & 4.79 & 4.7 & 1.59 & 3.39 & 1.73 & 0.67 & 0.13 \\
\hline 44 R & 1.11 & 1.98 & 1.5 & 1.44 & 1.59 & 0.35 & 1.15 & 0.4 & -0.05 & -0.05 \\
\hline 45 L & 4.61 & 6.28 & 4.84 & 4.84 & 4.76 & 1.62 & 3.42 & 1.75 & 0.69 & 0.15 \\
\hline 45 R & 1.13 & 1.97 & 1.5 & 1.43 & 1.6 & 0.37 & 1.17 & 0.41 & -0.03 & -0.02 \\
\hline 46 L & 2.44 & 3.44 & 2.35 & 2.46 & 2.17 & 0.42 & 1.57 & 0.51 & -0.17 & -0.37 \\
\hline 46 R & 1.2 & 2.13 & 1.51 & 1.48 & 1.52 & 0.39 & 1.19 & 0.45 & -0.01 & -0.05 \\
\hline 47 L & 4.82 & 6.16 & 4.67 & 4.54 & 4.68 & 1.77 & 3.56 & 1.93 & 0.83 & 0.27 \\
\hline $\mathbf{4 7 ~ R}$ & 1.12 & 1.89 & 1.35 & 1.22 & 1.47 & 0.38 & 1.15 & 0.41 & -0.02 & 0.03 \\
\hline la L & 4.64 & 5.09 & 3.14 & 2.84 & 3.24 & 1.65 & 3.19 & 1.93 & 0.8 & 0.23 \\
\hline la R & 1.72 & 2.19 & 1.12 & 0.93 & 1.27 & 0.48 & 1.25 & 0.58 & 0.08 & 0.05 \\
\hline us L & 4.6 & 4.78 & 2.75 & 2.46 & 2.84 & 1.61 & 3.02 & 1.95 & 0.82 & 0.24 \\
\hline us R & 1.81 & 2.15 & 1.04 & 0.86 & 1.2 & 0.52 & 1.24 & 0.66 & 0.12 & 0.04 \\
\hline & & & & & & & & & & \\
\hline
\end{tabular}

Figure 1. Z-scores greater than 3, which represent a deviation greater than 99\% are highlighted in red. The Brodmann Areas identified are all associated with addiction and there appears to be significant normalization of brain activity in these regions after treatment with Lisdexamfetamine dimesylate (LDX). 
Fourier transformed (FFT) to obtain the absolute spectral power for each channel in the following frequency bands: delta $(2-3 \mathrm{~Hz})$; theta $(4-8 \mathrm{~Hz})$; alpha (8 $12 \mathrm{~Hz})$; low beta $(12-20 \mathrm{~Hz})$; high beta $(21-30 \mathrm{~Hz})$ and gamma $(30-40 \mathrm{~Hz})$ Channels were then averaged to see the overall changes in band power before and after.

\section{Discussion}

BED is described by the DSM-V as recurrent and uncontrollable episodes of compulsive eating with marked physical and psychological distress [3]. The interplay between the compulsive eating behaviour elements: habitual overeating, overeating to relieve a negative emotional state, overeating despite adverse consequences, and the DA, TAAR1, and 5-HT systems marks the psychobehavioural and neuropharmacological mechanisms active in both BED and mechanisms of action by LDX [4]-[19].

In the present case, a patient with chronic BED, sought treatment after 45 years of ineffective surgeries, medications, and psychotherapy. After several LORETA neurofeedback sessions, the patient agreed to try LDX at $30 \mathrm{mg}$ a day for 6 months. Self-reported immediate cessations from binge-eating behaviours are accompanied by a normalization of brain areas associated with BED and addiction demonstrated by EEG scans pre- and post-treatment.

Immediately after beginning treatment with LDX, the patient reported cessation of binge-eating episodes and behaviours. Compared to the EEG scans taken before LDX treatment, post-LDX treatment scans showed a normalization of EEG activity in the Brodmann areas associated with BED and addiction. These Brodmann areas, 13, 24, 25, 32, 44, 45, 46, 47, include the insular cortex and PFC pathways, which are integral to executive functioning and inhibitory control in humans [21]. These areas participate in varying degrees to the craving, bingeing, and withdrawal behaviours seen in addiction [21] [22]. The advantage to using $\mathrm{Z}$-scores in LORETA biofeedback is the ability to receive instant comparisons using a reference database of healthy individuals Z-scores [23]. These instantaneous comparisons make it possible to see the link between patients' symptoms and the Brodmann areas implicated [23] [24]. Deficits in the PFC regions of the dlPFC (Brodmann area 46), ACC (Brodmann areas 24, 25, 32), right inferior frontal gyrus overlapping with the OFC (Brodmann area 47), and vmPFC (Brodmann areas 44,45), are thought to be at the route of dysfunction in compulsive eating behaviours and BED [8] [9] [21]. Along with the patient's self-reported cessation of all binge-eating behaviours and episodes, it is clear that the regions of her brain associated with BED have also normalized post-treatment as seen in Figure 1.

The F8 electrode corresponding to Brodmann area 47, an area associated with deficits in inhibitory function seen in addictions and BED [8] [9] [21], was also found to show a significant decrease in theta band power in the patient after treatment with LDX. Previous studies have shown significant modulation of 
30

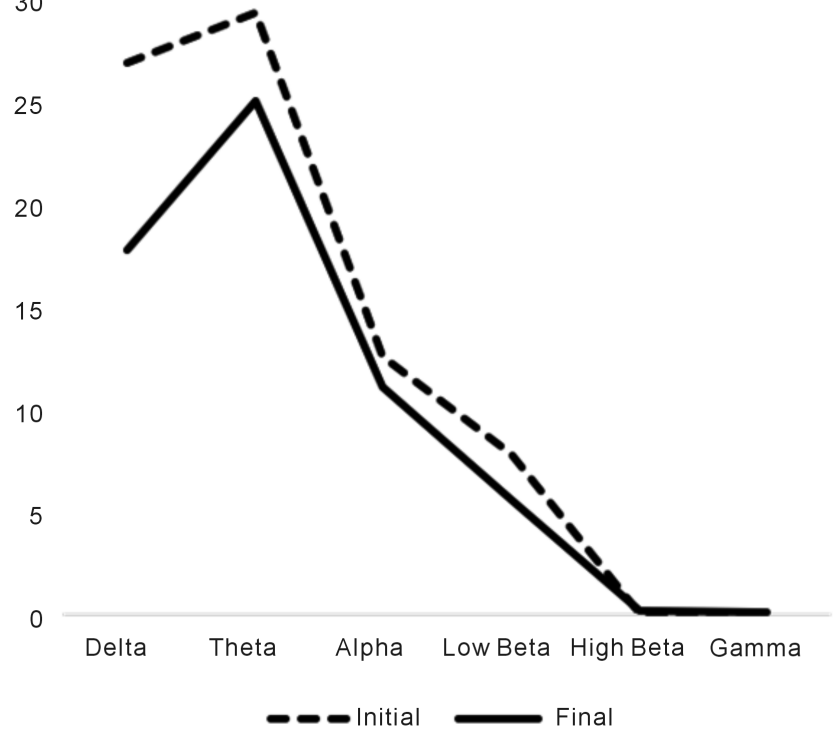

(a) power in the theta band of individuals with food or drug addiction [25] [26] [27] [28]. The power spectrum density of the patient shows that theta band power was significantly higher before treatment as shown in Figure 2. Several studies have shown that alcoholics exhibit increased overall power in the theta band relative to controls, hypothesized to be an index of imbalance in cortical excitation-inhibition homeostasis [25] [26].

Tammela et al., have also found some overlap in resting state EEG frequency bands between alcoholics and subjects with BED [27]. Further analysis shows that the change in theta power is driven primarily by a decrease of power in the right inferior frontal gyrus after treatment (BA 47 or F8 electrode) as shown in Figure 3. The right inferior frontal gyrus which overlaps with the OFC, is an area associated with deficits in inhibitory function seen in addictions and BED [8] [9]. In a study done by Imperatori et al., patients with three or more food addiction symptoms showed increased theta power in the right inferior frontal gyrus (BA 47 or F8) [28].

LDX, similar to other amphetamine-based medications, is a categorized Schedule II controlled medication, which contains a black box warning reporting its potential for abuse and dependence [1] [7] [19]. Prior to prescription, bipolar disorder evaluation and risks for abuse are required to be assessed, and throughout treatment, adverse psychiatric reactions, and signs of abuse and dependence monitored continually [1] [7] [19]. The safety profile for LDX in those with BED was found to be consistent with the adverse reactions seen in adults with ADHD [1] [19]. The most commonly reported adverse reactions for those
$31 \quad(b)$

30

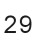

28

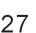

26

25

24

23

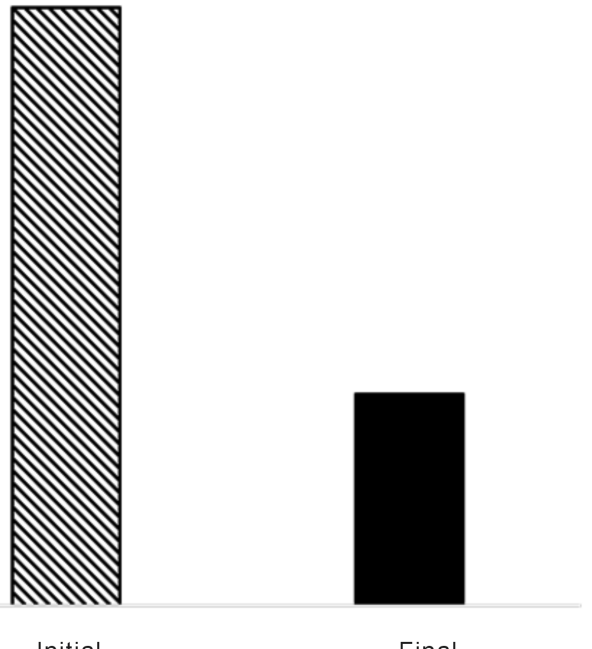

Figure 2. (a) The average power decreases in higher frequency bands, and there is a significant difference between delta and theta bands. (b) A closer look at the decrease in power in the theta band. The dashed lines represent the initial condition, and solid fill represents 3 months post treatment. 


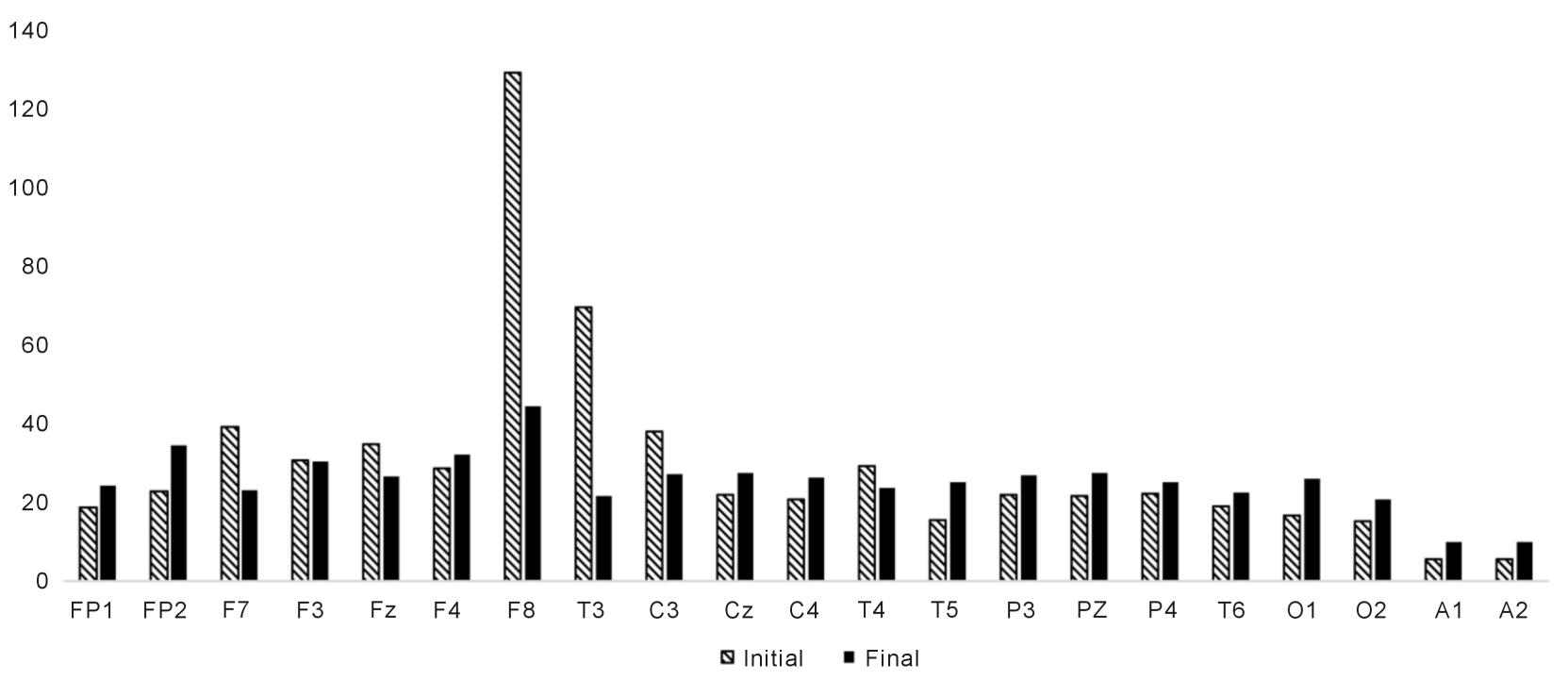

Figure 3. BA 47 or F8 electrode has the most significant decrease in power following treatment with Lisdexamfetamine dimesylate (LDX). The dashed lines represent the initial condition, and solid fill represents 3 months post-treatment.

with BED, also listed on the LDX prescription insert, include: dry mouth, headache, insomnia, decreased appetite, feeling jittery, anxiety, and increased heart rate and blood pressure [1] [7] [19].

In line with the medications reported warnings and adverse reactions, the patient reported dry mouth and lips, increased perspiration, and a need to take her dose of LDX by early morning to avoid an inability to sleep. The patient continues to report a complete normalization of binge-eating behaviours and still actively uses LDX for treatment.

Implications from this review and case report highlight brain regions implicated in BED and mechanisms of LDX action. Direct mechanisms of LDX and BED should be further studied in order to better understand the psychobehavioural and neuropharmacological mechanisms involved, and to enhance knowledge of the underlying pathogenesis, as well as ideal treatment options for those with BED. Future studies may also explore the effects of LDX on the theta power band frequency in those with BED.

\section{References}

[1] McElroy, S.L., Hudson, J.I., Mitchell, J.E., Wilfley, D., Ferreira-Cornwell, M.C., Gao, J., Wang, J., Whitaker, T., Jones, J. and Gasior, M. (2015) Efficacy and Safety of Lisdexamfetamine for Treatment of Adults with Moderate to Severe Binge-Eating Disorder: A Randomized Clinical Trial. JAMA Psychiatry, 72, 235-246.

https://doi.org/10.1001/jamapsychiatry.2014.2162

[2] Kessler, R.M., Hutson, P.H., Herman, B.K. and Potenza, M.N. (2016) The Neurobiological Basis of Binge-Eating Disorder. Neuroscience and Biobehavioral Reviews, 63, 223-238. https://doi.org/10.1016/j.neubiorev.2016.01.013

[3] American Psychiatric Association DSM-5 Task Force (2013) Diagnostic and Statistical Manual of Mental Disorders: DSM-5. 5th Edition, American Psychiatric Publishing, Washington, DC. 
[4] McElroy, S.L., Guerdjikova, A.I., Mori, N. and Keck Jr., P.E. (2015) Psychopharmacologic Treatment of Eating Disorders: Emerging Findings. Current Psychiatry Reports, 17, 1-7. https://doi.org/10.1007/s11920-015-0573-1

[5] Goodman, D.W. (2010) Lisdexamfetamine Dimesylate (Vyvanse), a Prodrug Stimulant for Attention-Deficit/Hyperactivity Disorder. $P \&$ T: A Peer-Reviewed Journal for Formulary Management, 35, 273.

[6] Guerdjikova, A.I., Mori, N., Blom, T.J., Keck, P.E., Williams, S.L., Welge, J.A. and McElroy, S.L. (2016) Lisdexamfetamine Dimesylate in Binge Eating Disorder: A Placebo Controlled Trial: Lisdexamfetamine in BED. Human Psychopharmacology: Clinical and Experimental, 31, 382-391. https://doi.org/10.1002/hup.2547

[7] Citrome, L. (2015) Lisdexamfetamine for Binge Eating Disorder in Adults: A Systematic Review of the Efficacy and Safety Profile for This Newly Approved Indication: What Is the Number Needed to Treat, Number Needed to Harm and Likelihood to Be Helped or Harmed? International Journal of Clinical Practice, 69, 410-421. https://doi.org/10.1111/ijcp.12639

[8] Moore, C., Panciera, J.I., Sabino, V. and Cottone, P. (2018) Neuropharmacology of Compulsive Eating. Philosophical Transactions, 373.

https://doi.org/10.1098/rstb.2017.0024

[9] Moore, C.F., Sabino, V., Koob, G.F. and Cottone, P. (2016, 2017) Pathological Overeating: Emerging Evidence for a Compulsivity Construct. Neuropsychopharmacology, 42, 1375-1389. https://doi.org/10.1038/npp.2016.269

[10] Cottone, P., Sabino, V., Roberto, M., Bajo, M., Pockros, L., Frihauf, J.B. and Zorrilla, E.P. (2009) CRF System Recruitment Mediates Dark Side of Compulsive Eating. Proceedings of the National Academy of Sciences of the United States of America, 106, 20016-20020. https://doi.org/10.1073/pnas.0908789106

[11] Parylak, S.L., Koob, G.F. and Zorrilla, E.P. (2011) The Dark Side of Food Addiction. Physiology \& Behavior, 104, 149-156. https://doi.org/10.1016/j.physbeh.2011.04.063

[12] Volkow, N.D., Wang, G. and Baler, R.D. (2011) Reward, Dopamine and the Control of Food Intake: Implications for Obesity. Trends in Cognitive Sciences, 15, 37-46. https://doi.org/10.1016/j.tics.2010.11.001

[13] Rae, C.L., Hughes, L.E., Anderson, M.C. and Rowe, J.B. (2015) The Prefrontal Cortex Achieves Inhibitory Control by Facilitating Subcortical Motor Pathway Connectivity. The Journal of Neuroscience: The Official Journal of the Society for Neuroscience, 35, 786-794. https://doi.org/10.1523/JNEUROSCI.3093-13.2015

[14] Furlong, T.M., Jayaweera, H.K., Balleine, B.W. and Corbit, L.H. (2014) Binge-Like Consumption of a Palatable Food Accelerates Habitual Control of Behavior and Is Dependent on Activation of the Dorsolateral Striatum. The Journal of Neuroscience: The Official Journal of the Society for Neuroscience, 34, 5012-5022. https://doi.org/10.1523/JNEUROSCI.3707-13.2014

[15] Ferragud, A., Howell, A.D., Moore, C.F., Ta, T.L., Hoener, M.C., Sabino, V. and Cottone, P. (2016, 2017) The Trace Amine-Associated Receptor 1 Agonist RO5256390 Blocks Compulsive, Binge-Like Eating in Rats. Neuropsychopharmacology, 42, 1458-1470. https://doi.org/10.1038/npp.2016.233

[16] Xu, P., He, Y., Cao, X., Valencia-Torres, L., Yan, X., Saito, K., Wang, C., Yang, Y., Hinton Jr., A., Zhu, L., Shu, G., Myers Jr., M.G., Wu, Q., Tong, Q., Heisler, L.K. and $\mathrm{Xu}, \mathrm{Y}$. (2016) Activation of Serotonin 2C Receptors in Dopamine Neurons Inhibits Binge-Like Eating in Mice. Biological Psychiatry, 81, 737-747.

https://doi.org/10.1016/j.biopsych.2016.06.005 
[17] Steiger, H. (2004) Eating Disorders and the Serotonin Connection: State, Trait and Developmental Effects. Journal of Psychiatry \& Neuroscience: JPN, 29, 20-29.

[18] Milano, W., Petrella, C., Casella, A., Capasso, A., Carrino, S. and Milano, L. (2005) Use of Sibutramine an Inhibitor of the Reuptake of Serotonin and Noradrenaline, in the Treatment of Binge Eating Disorder: A Placebo-Controlled Study. Advances in Therapy, 22, 25-31. https://doi.org/10.1007/BF02850181

[19] Guerdjikova, A.I., Mori, N., Casuto, L.S. and McElroy, S.L. (2016) Novel Pharmacologic Treatment in Acute Binge Eating Disorder: Role of Lisdexamfetamine. Neuropsychiatric Disease and Treatment, 12, 833-841. https://doi.org/10.2147/NDT.S80881

[20] Rowley, H.L., Kulkarni, R., Gosden, J., Brammer, R., Hackett, D. and Heal, D.J. (2012) Lisdexamfetamine and Immediate Release D-Amfetamine: Differences in Pharmacokinetic/Pharmacodynamic Relationships Revealed by Striatal Microdialysis in Freely-Moving Rats with Simultaneous Determination of Plasma Drug Concentrations and Locomotor Activity. Neuropharmacology, 63, 1064-1074. https://doi.org/10.1016/j.neuropharm.2012.07.008

[21] Goldstein, R.Z. and Volkow, N.D. (2011) Dysfunction of the Prefrontal Cortex in Addiction: Neuroimaging Findings and Clinical Implications. Nature Reviews Neuroscience, 12, 652-669. https://doi.org/10.1038/nrn3119

[22] De Ridder, D., Manning, P., Leong, S.L., Ross, S., Sutherland, W., Horwath, C. and Vanneste, S. (2016) The Brain, Obesity and Addiction: An EEG Neuroimaging Study. Scientific Reports, 6, Article No. 34122. https://doi.org/10.1038/srep34122

[23] Thatcher, R.W. (2010) LORETA Z Score Biofeedback. Neuroconnections, 9-13.

[24] Thatcher, R.W. and Lubar, J.F. (2015) Z Score Neurofeedback: Clinical Applications. Elsevier Inc., New York.

[25] Rangaswamy, M., Porjesz, B., Chorlian, D., Choi, K., Jones, K., Wang, K., Rohrbaugh, J., O'Connor, S., Kuperman, S., Reich, T. and Begleiter, H. (2003) Theta Power in the EEG of Alcoholics. Alcoholism-Clinical and Experimental Research, 27, 607-615. https://doi.org/10.1111/j.1530-0277.2003.tb04397.x

[26] Dymond, S., Lawrence, N., Dunkley, B., Yuen, K., Hinton, E., Dixon, M., Cox, W. M., Hoon, A.E., Munnelly, S.D., Muthukumaraswamy, K.D. and Singh, K. (2014) Almost Winning: Induced MEG Theta Power in Insula and Orbitofrontal Cortex Increases During Gambling Near-Misses and Is Associated with BOLD Signal and Gambling Severity. Neuroimage, 91, 210-219. https://doi.org/10.1016/j.neuroimage.2014.01.019

[27] Tammela, L.I., Paakkonen, A., Karhunen, L.J., Karhu, J., Uusitupa, M.I.J. and Kuikka, J.T. (2010) Brain Electrical Activity during Food Presentation in Obese Binge-Eating Women. Clinical Physiology and Functional Imaging, 30, 135-140. https://doi.org/10.1111/j.1475-097X.2009.00916.x

[28] Imperatori, C., Fabbricatore, M., Innamorati, M., Farina, B., Quintiliani, M.I., Lamis, D.A., Mazzucchi, E., Contardi, A., Vollono, C. and Della Marca, G. (2015) Modification of EEG Functional Connectivity and EEG Power Spectra in Overweight and Obese Patients with Food Addiction: An eLORETA Study. Brain Imaging and Behavior, 9, 703-716. https://doi.org/10.1007/s11682-014-9324-x 